\title{
Adhesion Measurement Methods for Thin Films in Microelectronics
}

\author{
Taek-Soo Kim*, $\dagger$ \\ *Department of Mechanical Engineering, KAIST \\ †Corresponding author : tskim1@kaist.ac.kr
}

\begin{abstract}
Diagnosing and improving adhesion of advanced packaging and thin films is an essential part of the research and development in microelectronics. In this paper, the four-point bend test and the double cantilever beam test among various adhesion measurement methods are reviewed.
\end{abstract}

Key Words : Adhesion, Fracture, Thin film, Microelectronics

\section{Introduction}

Thin films are ubiquitous and important in modern technology. For example, microelectronic devices, fuel cells, and solar cells, rely on a wide variety of advanced thin films. However, while significant efforts have been directed to improving electrical, optical, and electrochemical properties of thin films, mechanical integrity has been often neglected or in some cases even sacrificed. For example, low dielectric constant (k) insulating thin films for $\mathrm{Cu}$ interconnects in ultralarge scale integrated (ULSI) devices have achieved better dielectric properties by reducing film density. However, reducing film density resulted in greatly accelerated damage evolution in the form of delamination and cracking in low $k$ thin films during processes and in-service operation as shown in Fig. 1(a). Debonding at a Si/underfill interface by moisture assisted cracking and the mismatch of coefficient of thermal expansion (CTE) is also limiting further advances of microelectronic devices (Fig. 1(c)).

Therefore diagnosing and improving adhesion of advanced packaging and thin films is an

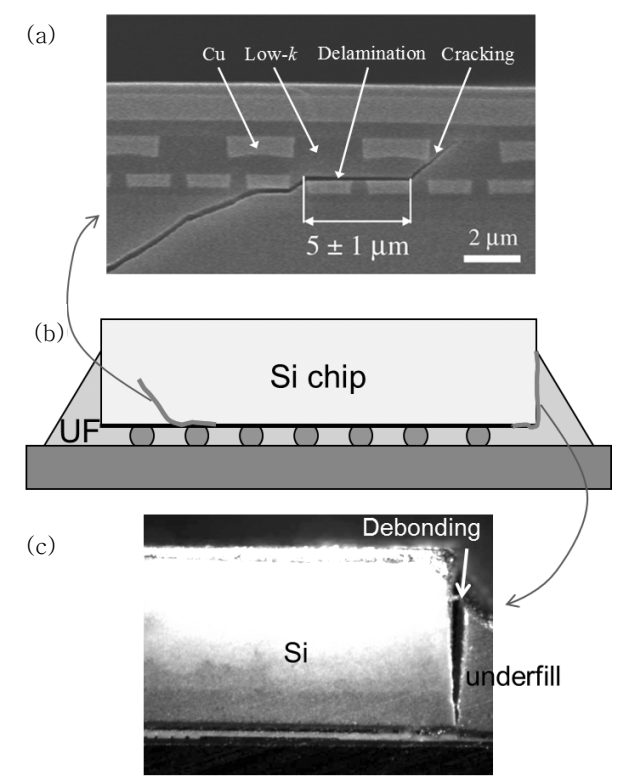

Fig. 1 (a) Cross-sectional image of $\mathrm{Cu} / \mathrm{low}^{-k}$ interconnects showing delamination and cracking in $l^{-}-k$ films ${ }^{1)}$. (b) Schematic illustration of a flip-chip packaging. (c) Cross-sectional image of a debonded $\mathrm{Si}$ / underfill interface

essential part of the research and development in microelectronics. Most adhesion measurement methods can be categorized into 1) peel test, 2) tape peel test, 3) indentation debonding test, 4) scratch test, 5) blister test, 6) self- 
loading test, and 7) beam-bending tests ${ }^{2)}$. Among these, beam-bending tests are based on quantitative analysis using fracture mechanics and mechanics of a bending beam, and suitable for the adhesion measurement of thin films deposited on $\mathrm{Si}$ wafers. In this paper, the four-point bend (FPB) test and the double cantilever beam (DCB) test among various beam-bending tests are reviewed.

\section{Fabrication of FPB and DCB Specimens}

An example of the fabrication of FPB and DCB specimens is as follows. In order to fabricate specimens for fracture testing, the thin-film structures of interest are sandwiched between elastic silicon substrates as shown in Fig. 2. The thin films of interest were initially deposited onto bare $\langle 100\rangle$ silicon wafers. These wafers are then cleaved along primary cleavage planes (along the $\langle 110\rangle$ type directions) into roughly $50 \mathrm{~mm} \times 50 \mathrm{~mm}$ sized coupons. The resulting structure is then bonded to a similarly sized coupon of bare silicon with a thin epoxy layer (EPO-TEK 353ND; Epoxy Technologies) using an epoxy bonding method ${ }^{3)}$. Once the specimens are bonded, a high speed wafer dicing saw is used to created specimens $\sim 3-5 \mathrm{~mm}$ in width.

Occasionally a poor surface finish associated with a damaged surface layer results in premature fracture of the substrates before the films debond. For structures that exhibit high fracture energies, further side surface preparation involving polishing of the side surfaces is

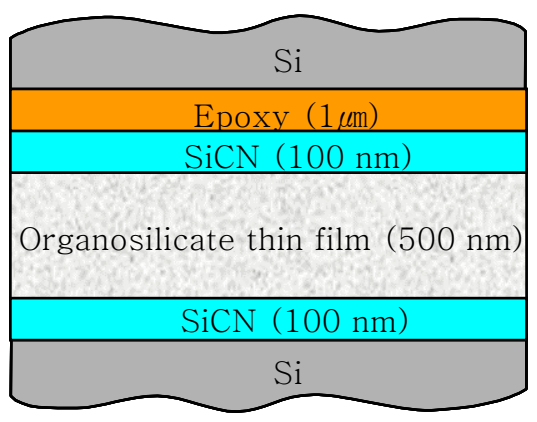

Fig. 2 Schematic of typical sandwiched thin-film structures required to prevent premature failure. In this event, the side faces of the specimens are ground and polished using a colloidal silica slurry with a final particle size of $\sim 20 \mathrm{~nm}$. This results in a near mirror finish which allows the specimens to be tested.

After dicing and polishing, aluminum loading tabs are attached to the DCB specimens using a high strength epoxy (DP-420, 3M, USA), and a schematic and an optical image of the DCB structure are shown in Fig. 3(a) and (c), respectively. A low-friction testing apparatus employing jeweled bearings in the loading tabs is employed. This enables subtle differences in crack growth behavior to be measured that may have otherwise been obscured in the presence of friction in the load train. The DCB specimens are then precracked to ensure that an initial crack of $\sim 5-10 \mathrm{~mm}$ is present prior to mechanical testing. The precracking is carried out using a small dicing saw (Buehler, Lake Bluff, IL) to create a small notch at the end of the specimen between the loading tabs. A wedge implement is then inserted into the notch in order to initiate the precrack. The FPB specimens are diced, polished in the same manner, and typically prenotched using a diamond scribe to create a line of damage across the upper silicon substrate (Fig. 3(b) and (d)). Alternatively, the pre-notch can be machined into the upper substrate to within $20 \mu \mathrm{m}$ of the thin-film interface using the high-speed diamond wafering blade. The FPB
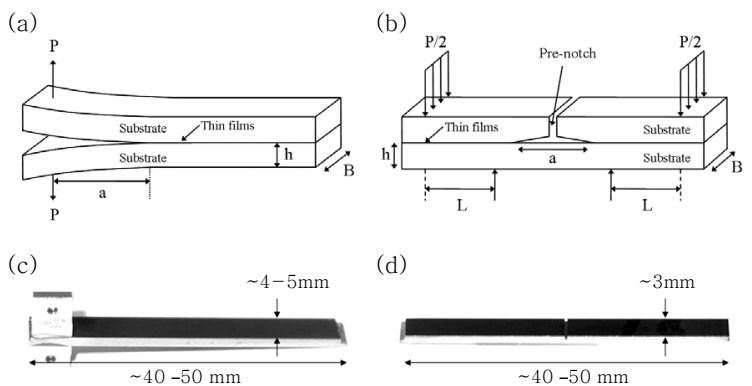

Fig. 3 Schematic illustration (a) and an actual image (c) of a DCB specimen. Schematic illustration (b) and actual image (d) of a FPB specimen 
specimen may be notched on either substrate, depending on which organosilicate thin film to barrier interface is to be investigated.

\section{Testing Techniques}

Critical fracture energy and subcritical crack growth measurements are conducted using a high-precision micromechanical test system (Delaminator Adhesion Test System; DTS Company, Menlo Park, CA) as shown in Fig. 4. Crosshead displacement was controlled with a servo actuator that featured a total displacement range of $50 \mathrm{~mm}$ and a displacement resolution of $50 \mathrm{~nm}$. The applied load is monitored with a $222 \mathrm{~N}$ load cell with a resolution of $0.01 \mathrm{~N}$. Typically critical fracture energy measurements are conducted in a lab air environment, and subcritical crack growth tests are conducted in an environmental chamber with the control of temperature and humidity. The aqueous solutions of interest for the subcritical crack growth tests are sealed in an inert Teflon container throughout the experiments to minimize evaporative losses and contamination of the solution. This is accomplished by submerging DCB specimen into the solution of interest by placing a Teflon container beneath the test frame as shown in Fig. 4.

\subsection{Four Point Bend Test}

The FPB geometry was initially developed to

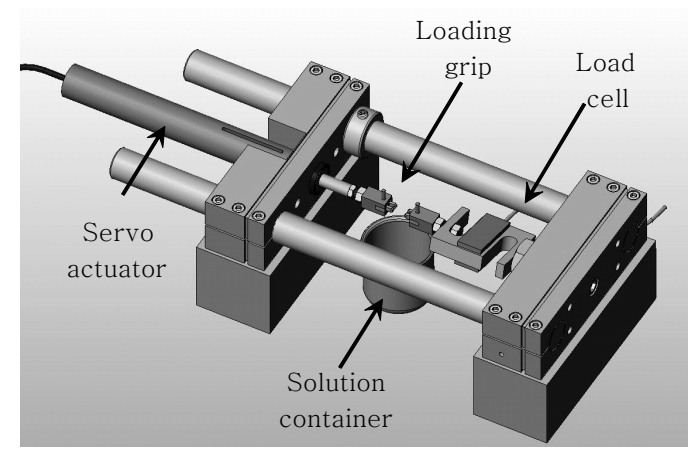

Fig. 4 The micromechanical test system used to conduct thin-film fracture energy measurements. The loading grips shown are for the double cantilever beam configuration study the adhesion of bimaterial interfaces ${ }^{4,5)}$, and it was later adapted to the study of thin-film multilayers ${ }^{6)}$. A typical four-point bend specimen is shown schematically in Fig 3(b). For the most common case of a symmetric specimen in which the upper and lower substrates have the same thicknesses and elastic properties, roughly equal amounts of normal and shearing stresses develop at the crack tip, resulting in a loading phase angle of $43^{\circ 4}$. This degree of mode mixity has relevance to a number of modern technological problems, including the debonding of thin films. Moreover, by varying the relative thicknesses and elastic moduli of the two substrates, a range of mixedmode conditions can be analyzed ${ }^{4)}$.

In order to run a four-point test, the specimen is loaded in four-point flexure at a constant crosshead displacement rate while the applied load is continuously monitored as a function of displacement (or time). A representative plot of load versus displacement is provided in Fig. 5. Initially, the composite structure loads elastically, storing strain energy within the substrates. At sufficient load, a crack emanates from the prenotch on the upper substrate, breaks through the upper substrate, and then bifurcates into the thin-film multilayer. The crack then extends through the multilayer, typically along some weak interface of interest, until it reaches the inner loading pins where crack growth arrests. During this fracture process, the applied load

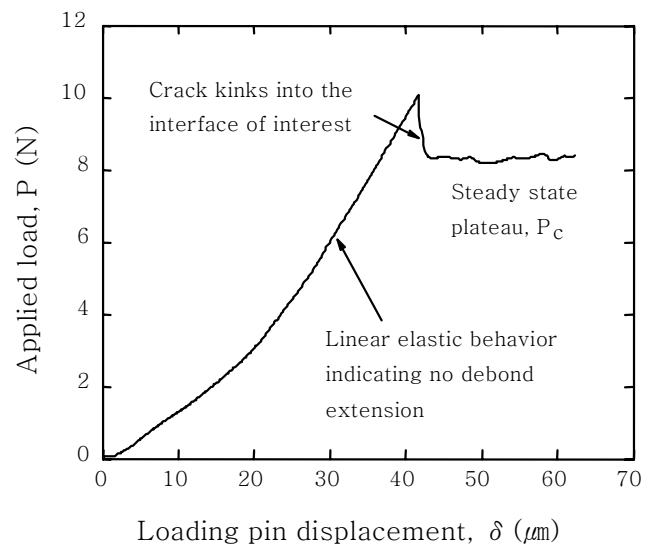

Fig. 5 Load versus displacement from a representative FPB test 
plateaus at a critical value, $P_{c}$. Based on very straightforward beam theory analysis, it can be shown that the critical strain energy release rate for a symmetric specimen is given by ${ }^{5)}$

$$
G_{c}=\frac{21 P_{c}^{2} L^{2}}{16 E^{\prime} B^{2} h^{3}}
$$

where $L$ is the applied moment arm, $E^{\prime}$ is the plane strain modulus of the substrate, $B$ is the specimen width, and $h$ is the specimen halfthickness (refer to Fig. 3(b)). The measured value of $G_{c}$ is used to report the fracture resistance of the interface that failed during the test. Note that the strain energy release rate does not depend on a, the crack length (which happens to be a direct consequence of the fact that the compliance of the four-point specimen is linearly related to crack length). Therefore it is not necessary to measure the crack length in order to measure fracture energy, which makes the four-point technique particularly simple to carry out in practice. The FPB technique is a very powerful method for studying near-interfacial fracture properties of thin films. Because of the mode-mixity involved with the loading configuration, the crack is typically driven to one of the interfaces of the thin film of interest. Moreover, by flipping the four-point specimen and pre-notching on the opposing substrate, fracture can be made to occur at the opposite of the thin film of interest to the adjacent layer interface. Thus the technique allows one to selectively probe both interfaces.

\subsection{Double Cantilever Beam Test}

The DCB geometry is often used to investigate fracture properties under nominally pure Mode I loading conditions ${ }^{7)}$. A typical symmetric double cantilever beam specimen is shown schematically in Fig. 3(a), and a representative plot of data from a double cantilever beam experiment is provided in Fig. 6. As with the four-point bend technique, the double cantilever

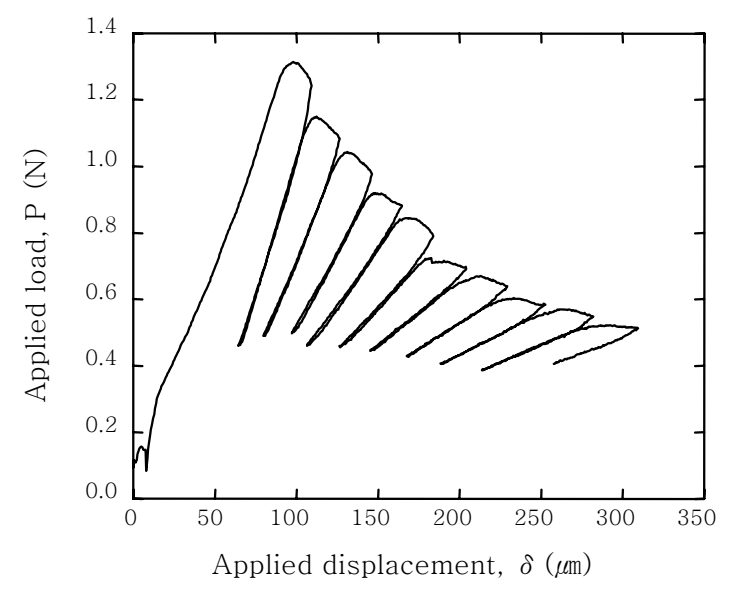

Fig. 6 Applied load versus displacement from a representative DCB critical test, displaying multiple loading/unloading cycles

beam specimen is loaded under constant displacement rate while the applied load is continuously monitored as a function of displacement. Initially, the specimen loads elastically, but once the strain energy release rate exceeds the fracture resistance of the layer or interface of interest, crack growth occurs within the thin-film multilayer. At this critical load $P_{c}$, the load versus displacement curve becomes non-linear, reflecting changes in specimen compliance as the crack extends. Following some increment of crack extension, the beam is partially unloaded and then subjected to multiple more loading/unloading cycles as shown in Fig. 6, in order to obtain multiple compliance curves from which the crack length can be accurately determined using the compliance-based relationship ${ }^{7)}$. Note that, unlike with four-point bend, the strain energy release rate of the double cantilever beam loading geometry depends on the crack length, a. The strain energy release rate can be calculated using the following formula ${ }^{7}$ :

$$
G_{c}=\frac{12 P_{c}^{2} a^{2}}{E^{\prime} b^{2} h^{3}}\left(1+0.64 \frac{h}{a}\right)^{2}
$$

Common practice for the double cantilever beam test is to report the critical fracture energy as the average of the critical fracture 
energies of the individual loading cycles.

Like four-point bend, the double cantilever beam technique is a powerful tool for the study of fracture behavior of thin films. Because the loading mode-mixity is nominally pure Mode I, fracture often proceeds near the middle of a weak film of interest (although fracture can also be interfacial if the interface is particularly weak). Thus the double cantilever geometry is very well suited to the study of cohesive fracture properties. As a result, it is very instructive to use four-point bend and double cantilever beam together for the study of fracture in thin films since they provide different loading phase angles and since one typically probes nearinterfacial properties whereas the other typically probes cohesive properties.

\subsection{Environmentally Assisted Crack Growth Experiments}

The sandwich flexural beam geometries are particularly well suited to the study of subcritical cracking of thin films. Both the FPB and DCB experimental setups can accommodate a wide range of environmental conditions, and the full crack growth rate versus strain energy release rate curve can be determined from a single controlled load relaxation experiment ${ }^{6)}$. The experiment involves loading a pre-cracked specimen just to the point of incipient crack growth and then fixing crosshead displacement. The load acting on the specimen is then measured as a function of time. During the experiment, the crack will extend over time due to the influence of the environment, and the load will relax with crack extension due to changes in specimen compliance. This is shown in Fig. 7. Straightforward compliance analyses are used to relate the crack growth rate at any given point to the time rate of change of the load, and these relationships are used to characterize the crack growth rate as a function of applied strain energy release rate throughout the test. For the FPB and DCB

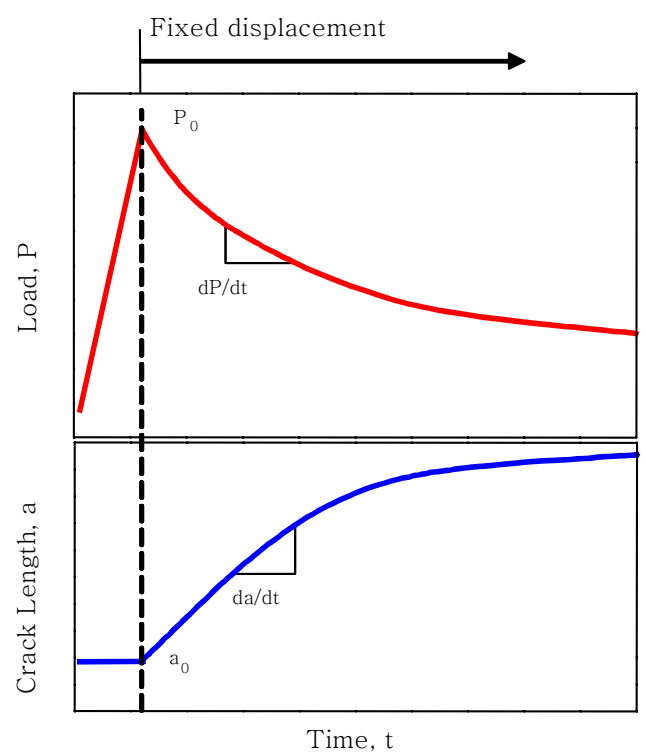

Fig. 7 Typical load relaxation data during a subcritical cracking experiment. The specimen is loaded until the point of incipient crack growth at which point displacement is fixed. The load on the specimen relaxes over time due to progressive crack extension

geometries, respectively, the crack velocity is given by

$$
\begin{aligned}
& \frac{d a}{d t}=-\frac{P_{0} a_{0}}{P^{2}}\left(\frac{d P}{d t}\right) \\
& \frac{d a}{d t}=-\frac{P_{0}^{1 / 3} a_{0}}{3 P^{4 / 3}}\left(\frac{d P}{d t}\right)
\end{aligned}
$$

where ao and $P_{0}$ are the initial crack length and load and $P$ is the instantaneous load.

\section{Conclusions}

In this paper, the four-point bend test and the double cantilever beam test among various adhesion measurement methods are reviewed.

\section{Acknowledgement}

This research was supported by Basic Science Research Program through the National Research Foundation of Korea (NRF) funded by the Ministry of Education, Science and Technology (N01120349). 


\section{References}

1. Ocaña, I., et al., Fracture characterization in patterned thin films by cross $^{-}$sectional nanoindentation. Acta Mat., 2006. 54(13), 3453-3462

2. Lacombe, R., Adhesion Measurement Methods, 2006 , CRC Press

3. Hohlfelder, R.J., et al., Adhesion of benzocyclobutenepassivated silicon in epoxy layered structures. Journal of Materials Research, 2001. 16(1), 243-255

4. Charalambides, P.G., et al., Development of a test method for measuring the mixed mode fracture resistance of bimaterial interfaces. Mechanics of Materials, 1990. 8(4), 269-283

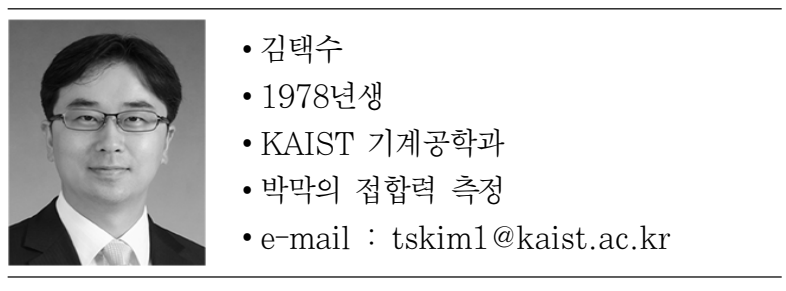

5. Charalambides, P.G., et al., A Test Specimen for Determining the Fracture Resisitance of Bimaterial Interfaces. Journal of Applied Mechanics, 1989. 56(1), $77-82$

6. Dauskardt, R.H., et al., Adhesion and debonding of multi-layer thin film structures. Engineering Fracture Mechanics, 1998. 61, 141-162

7. Kanninen, M.F., An augmented double cantilever beam model for studying crack propagation and arrest. International Journal of Fracture, 1973. 9(1), 83-92 